\title{
The Disappearance of the Screen Research on Audible Interfaces in the Ubiquitous Computing Environment
}

\author{
Daniel Michelis, Thomas Nicolai \\ University of St. Gallen \\ thomas.nicolai@unisg.ch \\ michelis@ieb.net
}

\author{
Florian Resatsch, Thomas Schildhauer \\ University of the Arts Berlin \\ resatsch@ieb.net \\ schildhauer@ieb.net
}

\begin{abstract}
The world of ubiquitous computing, which by definition includes mobile devices of every kind, leads us to an era of small computer devices, usable in everyday situations. Computers are becoming smaller and operate discreetly in the background. This paper deals with the disappearance of screen that is described and specified according to Lev Manovich. In doing research on radio frequency identification (RFID), this paper shows one possible way to interact with ubiquitous computers - primarily exploring audible interfaces. The paper describes a research project of the University of Arts Berlin and the University of St. Gallen and proposes future research questions.
\end{abstract}

\section{Introduction}

The digital network currently finds itself on the verge of entering a new dimension. It is a dimension circumscribed by the buzz words Ambient Intelligence, Ubiquitous or Pervasive Computing. Everything, every area of life down to the smallest aspect is being computerized and linked. Computers are becoming smaller and operate discreetly in the background. Through the ubiquity of the digital network, the relationship between man and computer is being redefined [1]. Why is this paradigm so important, even today with focus on mobile business? To quote Weiser: "The most profound technologies are those that disappear. They weave themselves into the fabric of everyday life until they are indistinguishable from it." [1] The mobile world today is approaching a scenario of everyday everywhere availability. Nevertheless, the interaction between humans and devices is still based on various forms of screens. But for a ubiquitous integration into our everyday life, new ways of interaction are necessary.

New forms of navigation in the world of Ubiquitous Computing are the major focus of this research paper.
There are currently many visionary scenarios of ubiquitous and pervasive computing, but most of them focus on the future rather than taking into consideration the issues raised by the immanent and pervasive use of mobile communication. These include issues about the interaction between humans and machines but also between devices. What does this relationship look like, and how will the interaction between humans and computers develop in the next few years? In search of possible answers, Manovich's "Language of New Media" offers worthwhile suggestions [2]. In his seminal theory of new media, also regarded as the most exact definition of new media, a list of concrete examples on the future of the interface between humans and computers is laid out [3]. According to Manovich, automation and the stepby-step disappearance of the screen brings with it noticeable repercussions to established visual forms of reality simulation. The dominance of the screen, that for hundreds of years shaped the simulation of reality, is being displaced by new forms of interaction. Multifunctional human computer interfaces herald the end of the screen era. For realistic representations the simple visualization of media content is no longer sufficient. With the disappearance of the screen the computer increasingly responds to the behaviour of the user. It acts and reacts, grows, moves and develops on the go, it even thinks and feels and, in the process, gives rise to a complex picture of a new reality that appears more real. Within this context the area of research of Ubiquitous Computing ponders the question: which human computer interfaces related to screen interactions may be set aside? Through which mechanisms, which technologies and through which senses are humans integrated into the future world of the computer? In short, what language do we speak to the computer after the disappearance of its primary interface: the screen? With respect to the example of the currently hyped radio frequency identification (RFID) technology, this research paper tries to develop 
insight into the future world of interaction between humans and computers.

This paper is structured into two main parts. In section two, the disappearance of the screen is described and specified according to Lev Manovich. Section three deals with the hypertagging project that was conducted by researchers at the University of Arts in Berlin. Originally using RFID technology, hypertagging shows one possible way to interact with (ubiquitous) computers — primarily providing an audible interface. In the conclusion, we try to integrate the implications of the hypertagging project into the vision of Lev Manovich.

\section{The Disappearance of the Screen}

In an attempt to analyze the historical development of new media in the context of traditional media, Manovich primarily takes stock of the situation. He is searching for an all over logic, with which to observe conventions and repeating patterns in order to explain the essential characteristic forms of new media. Manovich designates this logic as the language of new media. After Warner, Manovich provided with his work the heretofore most precise definition of new digital media, a definition that classifies the broadest historical media context since Marshall McLuhan [3]. In asking what makes new digital media different from old media Warner refers to a group of researches, who have approached the task from varying disciplines ranging from the history of technical culture (J. David Bolter) [4], to hypertext (George P. Landow) [5], theatre (Brenda Laurel) [6], narrative (Janet Murray) [7], architecture (William J. Mitchell) [8], and virtual reality (Michael Heim). The theories of these authors describe a range of general characteristics of new media as somewhat procedural, participatory and spatial (Murray) or discreet, conventional, finite and isolated (Bolter), that unite however around particular rather than holistic and general analysis. Necessary rather, is the isolation of essential new media characteristics, since the attempt to define general aspects is fraught with a fundamental problem: whoever ascribes new media characteristics that derive from the old media standards, risks downplaying the new and the difference of new media. Manovich's principles of new media numerical representation, modularity, automation, variability, and transcoding built on the starting point of his own theories, regard Warner as an initial felicitous point of departure to a solution of this dilemma [3].

The term human-computer-interface describes the way in which we are integrated with the computer. Next to the graphics on the screen and the metaphorical navigations, this terminology also circumscribes the hardware such as keyboard and mouse. This interface exemplifies the way we access and manipulate computer data. For Manovich, the shifting properties between computer and cultural realms are easily observed within this interface. Only a few years ago computers were still basic tools used to create and produce simple cultural content. Accordingly, the contents of the end product were tailored to the characteristic media: print, film, photo, and sound. Since the end of the 1990's, the role of the computer in society has clearly changed. It has been transformed from a basic tool into a universal media machine, not only to produce media content but to save and consume it as well. Manovich takes this a step further, when he asserts that all forms of culture today are based on the computer. Even the content of traditional media has increasingly adopted human computer interfaces that in turn increasingly impact the nature of the entire human culture. Accordingly, society "interfaces" cross-over media and culture in increasing amounts [2].

While the multi-layered functions of the computer were made possible by modern computer technologies, they were first brought to life through a very old technology: the screen. At the first glance of the screen, the illusion arises within the viewer that he finds himself in a bookstore or that he is moving through virtual worlds. In opposition to the computer, which has only become common in the last ten years, the screen has been used for hundreds of years to present visual information- from early painting to 20th century cinema. With the global linking of computers over the internet any type of information is received on the screen: Pictures, films, newspapers, news from friends and co-workers. With this latest incarnation of the screen our society has transformed into a society of the screen. In the developmental history of the screen Manovich differentiates between three essential phases that he designates as classic screen, dynamic screen, and real-time screen. During all three phases the screen is characterized by the existence of another virtual place, a three-dimensional world enclosed within its frame. Via this frame two totally different worlds are separated from one another, and yet exist simultaneously parallel to one another. The classic screen phase begins with painting on canvas and lasts until today: elements of the classic screen can be found on modern computers. As a flat, four-cornered surface, the screen simulates a special reality. Approximately one hundred years ago with the development of the moving image, the dynamic screen phase began. While in its new form it still retains all the characteristics of the classic screen, one central function is widened: the dynamic screen can display images that change with the passing of time. Manovich also designates the 
dynamic screen as the screen of cinema, televisions and video. With the spread of the dynamic screen the relationship between screen and viewer is altered. A new viewing regime takes hold: since the image of the dynamic screen simulates a possible total reality, the viewer identifies with the image and gives into the illusion. Hence in the new viewing regime the screen takes on the active roll, insofar as it makes possible much of the filtering out and blending out of what takes place outside its frame. The third phase, the phase of the real-time screen that also corresponds to the introduction of the computer screen, amounts to a fundamental reordering of the relationship between viewer and screen. With the introduction of the realtime screen, the viewer is pulled out of his passivity and becomes an active user. Thus, the computer screen isn't limited to the display of a single image. The display of windows arranged on top of one another with an entire range of diverse images becomes a basic principle of the real-time screen. The arrangement of different images and compositions take the user over. One no longer focuses on the single image, instead one steers the simulation on the screen independently and takes an active roll in the construction of one's subjective reality. While from the passive viewer a more active, more reflective and goal-oriented user has developed, all three forms of the screen still exist side by side. Each screen simulates accordingly the manner inherent in it and its specific reality and makes possible a determined spectrum of interactions. With a view toward virtual reality and ubiquitous computing, Manovich asserts that the screen in the future slowly disappears. When the simulated and the real world fuse together the screen connecting the two becomes redundant. The virtual world was in the past simulated by painting or film, now it becomes increasingly integrated into the actual, physical world itself [2].

With the disappearance of the screen, traditional principles of reality simulation and typical patterns of reception are lost. In a world of constant digital networks, in which the computer increasingly eludes visual perception, new forms of interaction will develop. Computers will speak to us in a new language and explain their histories according to new rules.

Next to the meaning of the screen and the impact of its gradual disappearance, there is another aspect from Manovich's theory related to the disappearance of screen: interactivity. For the simulation of reality the basic visualization of information is no longer sufficient: multi-functional human computer interfaces supersede the screen era. With the successive disappearance of the screen the computer orients itself and its interface increasingly to the behaviour of the user. The new formula reads: the smaller the interface, the more real the illusion. Insofar as computers act, react, grow, move around and develop over time, think and even feel, they give rise to a complex picture of a new meta-reality. The quest after less interface is not the pursuit of less control. The viewer is emancipated and intelligent and knows learns quickly how to interact with its surrounding.

\section{RFID Research Project hypertagging}

The hypertagging project is an interactive, qualitative research project conducted by the Institute of Electronic Business at the University of Arts Berlin, Germany, in conjunction with the Institute of Media and Communication Management at the University of St. Gallen, Switzerland. Hypertagging is based on RFID-tags, which can be attached to or embedded in any kind of objects. In order to explore central research questions in the emerging field of RFID-based humancomputer-interaction, the project will be carried out by the affiliated institutions as an ongoing ethnographic field study until 2007. In the near future, RFID tags will identify most commercial items. Stickers that will record, link or store all information that companies need to know about manufacture, ownership, value and origin. By placing RFID technology in the context of human-computer-interaction, the hypertagging project follows a different approach than most RFID research. Due to the artistic exploration of RFID technology and the integration of individuals, hypertagging is looking for potential RFID applications at the interface between technology, business and society.

\section{RFID-technology}

A RFID reader sends out a radio frequency wave to the "Tag" and the tag broadcasts back its stored data to the reader. The system works basically as two antennas, one on the tag and the other on the reader [9]. There are two different types of tags, active and passive ones, the passive ones which are battery-free currently play the major role in most RFID scenarios. Although known for more than sixty years, the potential of RFID technology was first recognized in the 1990s, mainly stimulating the desire for RFID-supported applications such as product tracking, logistics in general and inventory and counterfeit control [10]. Besides these areas, passive RFID tags are suited to augmenting physical objects, bridging the gap between physical and virtual worlds - the paradigm of Ubiquitous Computing environments [11]. The proliferation of RFID technology is advancing rapidly, while RFID reader devices, antennas and tags are becoming increasingly smaller and cheaper. As a result, the deployment of RFID technology on a larger scale is 
about to become both technically and economically feasible. In the conventional process of RFID tag deployment prevailing today, only a limited number of passive tags are placed in the environment in a deliberate and sparse fashion. RFID tags are mainly used for identifying objects and for detecting the containedness relationships of these objects [12]. Explicitly placed stationary tags embedded in the environment can also serve as dedicated artificial landmarks. They can be detected by means of a mobile RFID reader and are used to support the navigation of mobile devices and robots [13,14], or to mark places and passageways [15]. By doing this, the gap between the physical and virtual world is getting smaller. Still, there is no evidence that the distribution and the usage of the tags in public space, buildings or any other physical area itself will bring the ubiquitous computing paradigm to the desired level.

\section{Screen-based Sub-projects}

The hypertagging project tries to use RFID-technology as a new way of human-computer interface. Two hypertagging sub-projects have already been realised in the past. In July 2004, the sub-project data carrier was realized at a networking event of the Berlin Senate at the German Historical Museum in Berlin, Germany. The focus of data carrier was to reverse the traditional roles. With RFID-tags, embedded in their invitation cards, guests became exhibits. As passive data carriers they unknowingly revealed personal information and movements to the audience.

In August 2004, the second sub-project was presented as part of the Social Fictions Conference at the 12th International Symposium of Electronic Arts (ISEA2004) in Helsinki, Finland [16].

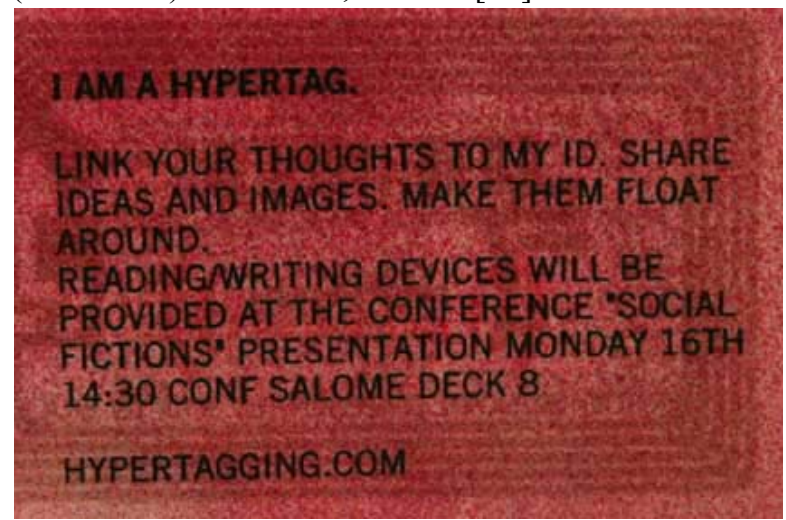

Under the title floating thoughts hypertags were used as communication media that could be used to transmit and share personal information (See picture above).

Observation and discussions with the participants of the sub-projects led to the further development of main research questions. Both installations were still using screen-based interfaces. With the disappearance of screen RFID-tags could become a new way to interact with the computer. Vice versa, as the following planed but not yet realised hypertagging sub-projects try to investigate, RFID could be part of new approach to simulate reality.

\section{Screen-less Sub-projects}

The sub-project "Attached Sounds" uses a simple framework that consists of long-range RFID-readers, high-performance microphones, a computer network and a couple of feedback speakers. Microphone and speakers serve as the audible interface between participant and computer.

To explore the impacts of this audible interface and to develop screenless ways of human-computer interaction the following hypertagging scenario is in development. Once the participant, who is tagged with "his" RFID-tag, enters the range of the RFID-reader the microphone starts recording. As long as the participant stays in reading-range the microphone records it's surrounding and links it to the participants RFID. All sound files in the database can be related not only to the participant but also to the time and space of its appearing.

The feedback speakers are placed in quiet environments where the participant can stay in physic (and acoustic) distance from others. It would even be possible to choose locations where the participant is alone by nature, e.g. a bathroom. Once he approaches the feedback speakers his RFID-tag will be identified and linked to the sounds that were recorded when he was close to the microphones.

The project Attached Sounds tries to explore one possible way of acoustic human-computer interactions. It rises questions of how people that are passively (and potentially not knowingly) interacting with a ubiquitous environment. How this relationship to technology develops over time and what needs and/or fears drive the acceptance of applications in other areas of public live such as administration or business is the question for further research.

\section{Subsonic Environments}

The second screenless sub-project that is planed to be carried out in 2005 is called sonic environment. Again, RFID-tags are attached to the participants. According to their status, history or project-role they have a certain sound that is explicitly associated to their person. Their sound is only one part of a whole sonic environment, be it an underwater environment, the shore or even the Australian jungle. Once a participant 
enters the reading range of the RFID-reader the audience will hear his sound, e.g. a breaking wave. Other members of the audience should get curious and start find out, that is, to hear their sound. If it corresponds to the same sonic environment, it will be layered over the previous sound(s). However, if it does not correspond it starts a new sonic environment.

Again, the research focus of this sub-project is to find out how people interact without a screen to invisible but ubiquitous computers. This would lead to a possible interaction scenario based on sounds in a museum or at the airport.

\section{Conclusion}

Through the increasingly ubiquitous availability of new media, the abundance of globally available information is supplemented by the mass of, up until now, non-digital location based information. The more it seems then in this context that the supply of information becomes an enormous challenge. At the same time, with the aforementioned retreat of the screen, new forms of the human computer interface are necessary that can speak to a greater range of human senses. By conducting research on screen-less humancomputer-interaction an important contribution to the future relationship between humans and computers in the era of ubiquitous computing can be achieved. With the interdisciplinary research project hypertagging, the authors try to explore acoustic navigation and sonic human-computer-interaction. The central (research) questions related to screen-less interaction in ubiquitous environments can be formulated in the language of new media as follows: What challenges must the human-computer interface satisfy after the end of the screen era?

What significance does the simulation of reality have and what rules govern the creation of illusions? If the visual presentation of information and the significance of the graphical interface are reduced, through which senses will humans be integrated with the computer in the future? How does the user's way of receiving information influence the development of interaction in the world of ubiquitous computing? How can immaterial interfaces assist the user at work through narration? The hypertagging project is a first step to explore the future of ubiquitous computing by providing new insights to screen-less humancomputer-interaction that will become more and more important as part of mobile applications in the ubiquitous computing world. The success and the acceptance of the users show a growing need in future screen-less applications.

\section{References}

[1] Weiser, M (1991), The Computer for the $21^{\text {st }}$ Century, http://www.ubiq.com/hypertext/weiser/SciAmDraft3.html

[2] Manovich, Lev (2001), The Language of New Media, MIT Press, Cambridge, Mass.

[3] Warner, William B. (2001), Review Computable Culture and the Closure of the Media Paradigm: Lev Manovich's The Language of New Media, UCSB Review

[4] Bolter, J.David (1999), Remediation: Understanding New Media, MIT Press, Cambridge, Mass.

[5] Landow, George P. (1994), Hyper/Text/Theory, Johns Hopkins University Press

[6] Laurel, Brenda (1993), Computers as Theatre, AddisonWesley

[7] Murray, Janet (1998), Hamlet on the Holodeck, MIT Press, Cambridge, Mass.

[8] Mitchell, William J. (1996), City of Bits: Leben in der Stadt des 21. Jahrhunderts, Birkhäuser, Basel

[9] http://www.rf-id.com/rfidtech.htm. Acc. Feb. 16, 2005

[10] J.R. Tuttle, Traditional and emerging technologies and applications in the radio frequency identification (RFID) industry. In IEEE Radio Frequency Integrated Circuits (RFIC) Symposium, pages 5-8, June 1997.

[11] R. Want, K. P. Fishkin, A. Gujar, and Beverly L. Harrison, Bridging physical and virtual worlds with electronic tags. In Proc. SIGCHI Conference on Human Factors in Computing Systems, pages 370-377. ACM Press, 1999

[12] J. Bohn, F. Mattern, Super-Distributed RFID Tag Infrastructures. 2004

[13] M. Lampe and C. Floerkemeier. The Smart Box application model. In Alois Ferscha, Horst Hoertner, and Gabriele Kotsis, editors, Advances in Pervasive Computing, pages 351-356. Austrian Computer Society (OCG), 04.2004

[14] L.M. Ni, Y. Liu, Y.C. Lau, and A.P. Patil. Landmarc: indoor location sensing using active RFID. In Proc. 1st IEEE Int. Conference on Pervasive Computing and Communications 2003, pages 407-415, March 2003

[15] K. Goto, Matsubara H., and S. Myojo. A mobile guide system for visually disabled persons. In Proc. 4th Int. Symposium on Autonomous Decentralized Systems, pages 12-17, March 1999

[16] Michelis, Daniel/ Send, Hendrik, hypertagging: Floating Thoughts, Conference Social Fictions, ISEA2004 